\title{
Article
}

\section{High Expression of PPM1D Induces Tumors Phenotypically Similar to TP53 Loss-of-Function Mutations in Mice}

\author{
Jelena Milosevic ${ }^{1,2, *(\mathbb{D})}$, Susanne Fransson ${ }^{3} \mathbb{D}$, Miklos Gulyas ${ }^{4}\left(\mathbb{D}\right.$, Thale K. Olsen ${ }^{1}$, Gabriel Gallo-Oller ${ }^{1}$ (D), \\ Diana Treis ${ }^{1}$ (D), Lotta H. M. Elfman ${ }^{1}$, Margareta Wilhelm ${ }^{5}$, Tommy Martinsson ${ }^{3}$, Ninib Baryawno ${ }^{1}$, \\ Per Kogner ${ }^{1,+(\mathbb{D})}$ and John Inge Johnsen ${ }^{1, *,+}$
}

1 Childhood Cancer Research Unit, Department of Women's and Children's Health, Karolinska Institutet, 17177 Stockholm, Sweden; thale.kristin.olsen@ki.se (T.K.O.); ggallo@alumni.unav.es (G.G.-O.); diana.treis@ki.se (D.T.); lotta.elfman@ki.se (L.H.M.E.); n.baryawno@ki.se (N.B.); per.kogner@ki.se (P.K.)

2 Center for Regenerative Medicine, Massachusetts General Hospital, Boston, MA 02114, USA

3 Department of Laboratory Medicine, Institute of Biomedicine, University of Gothenburg, 41345 Gothenburg, Sweden; susanne.fransson@clingen.gu.se (S.F.); tommy.martinsson@clingen.gu.se (T.M.)

4 Department of Immunology, Genetics and Pathology, Uppsala University, 75185 Uppsala, Sweden; miklos.gulyas@igp.uu.se

5 Department of Microbiology, Cell and Tumor Biology, Karolinska Institutet, 17177 Stockholm, Sweden; Margareta.Wilhelm@ki.se

* Correspondence: jmilosevic@mgh.harvard.edu (J.M.); john.inge.johnsen@ki.se (J.I.J.)

+ Senior authors with equal contribution.

Citation: Milosevic, J.; Fransson, S.; Gulyas, M.; Olsen, T.K.; Gallo-Oller, G.; Treis, D.; Elfman, L.H.M.; Wilhelm, M.; Martinsson, T.; Baryawno, N.; et al. High Expression of PPM1D Induces Tumors Phenotypically Similar to TP53 Loss-of-Function Mutations in Mice. Cancers 2021, 13, 5493. https://doi.org/ 10.3390/cancers13215493

Academic Editors: Marc Ekker and Dong Kwon Yang

Received: 2 October 2021

Accepted: 27 October 2021

Published: 31 October 2021

Publisher's Note: MDPI stays neutral with regard to jurisdictional claims in published maps and institutional affiliations.

Copyright: (c) 2021 by the authors. Licensee MDPI, Basel, Switzerland. This article is an open access article distributed under the terms and conditions of the Creative Commons Attribution (CC BY) license (https:/ / creativecommons.org/licenses/by/ $4.0 /)$.
Simple Summary: Aberrant expression of the PPM1D gene which encodes a phosphatase called WIP1 is frequently observed in cancers of different origins. WIP1 is a negative regulator of the tumor suppressor p53. Improper inactivation of p53 results in genomic instability and can induce neoplastic transformation. We show that overexpression of PPM1D induces tumors in mice similar to cancers harboring p53 mutations. Our results suggest that PPM1D can act as an oncogenic driver by inducing genomic instability, impaired growth arrest, and apoptotic escape that can result in neoplastic transformation and malignant tumor development.

Abstract: PPM1D is a negative regulator of p53 and genomic aberrations resulting in increased activity of PPM1D have been observed in cancers of different origins, indicating that PPM1D has oncogenic properties. We established a transgenic mouse model overexpressing PPM1D and showed that these mice developed a wide variety of cancers. PPM1D-expressing mice developed tumors phenotypically and genetically similar to tumors in mice with dysfunctional p53. T-cell lymphoblastic lymphoma was the most frequent cancer observed in these mice (55\%) followed by adenocarcinomas $(24 \%)$, leukemia (12\%) and other solid tumors including neuroblastoma. Characterization of Tcell lymphomas in mice overexpressing PPM1D demonstrates Pten-deletion and p53-accumulation similar to mice with p53 loss-of-function. Also, Notch1 mutations which are recurrently observed in T-cell acute lymphoblastic lymphoma (T-ALL) were frequently detected in PPM1D-transgenic mice. Hence, PPM1D acts as an oncogenic driver in connection with cellular stress, suggesting that the PPM1D gene status and expression levels should be investigated in TP53 wild-type tumors.

Keywords: PPM1D; p53; genetically engineered mouse model; adenocarcinoma; lymphoma; neuroblastoma

\section{Introduction}

The protein phosphatase magnesium-dependent 1 delta $(P P M 1 D)$ gene encodes a nuclear serine/threonine phosphatase included in the PP2C family of phosphatases named WIP1 (wild-type p53-induced phosphatase 1) [1]. WIP1 is a critical regulator of DNA damage response and cell-cycle progression by its ability to regulate the activity of the tumor-suppressor protein p53, ATM, CHK1/2, and other key molecules involved in cell-cycle progression, DNA 
repair mechanisms, and apoptosis [2-7]. In concordance, PPM1D mutations, amplifications, gene fusions, and WIP1 overexpression have been observed in various cancers [8-17].

Inactivation of p53 is seen in more than $50 \%$ of human cancers and results in genomic instability, impaired growth arrest, and apoptotic escape allowing for continued cell proliferation and neoplastic induction [18,19]. p53 is induced and stabilized following DNA damage through multiple posttranslational modifications, ultimately leading to transcriptional activation of p53-target genes orchestrating cell-cycle progression, DNA repair mechanisms, and apoptosis [18]. In order to facilitate a rapid switch between an active and inactive state of p53, several mechanisms regulating the activity of p53 have been described. One important factor is the MDM2 oncogene encoding an E3 ubiquitin ligase [20,21]. MDM2 expression is induced by p53 which results in p53-MDM2 complex formation, MDM2-mediated ubiquitination, and proteasomal degradation of p53 [21]. This p53-MDM2 autoregulatory loop is controlled by ATM and ATR as well as other kinases that, immediately following DNA damage, phosphorylate MDM2 and p53 in a manner that inhibits the p53-MDM2 interaction leading to stabilization of p53 [22-24]. In order to restore the normal homeostatic state of the cell after completion of DNA repair, a set of phosphatases dephosphorylate p53 and MDM2 to allow for p53-MDM2 interaction and proteasomal degradation of p53. The expression level of WIP1 is controlled directly by p53 through binding to regulatory elements located in the $5^{\prime}$-untranslated region of the PPM1D gene $[1,25]$. WIP1 has been shown to dephosphorylate and inactivate p53 (Ser15) as well as the p53-activating kinases ATM, CHK1, and CHK2 that phosphorylate p53 at Ser15 and Ser20, respectively [2-7]. WIP1 also dephosphorylates MDM2 at Ser395, a site phosphorylated by ATM [26]. The dephosphorylation of p53 and p53-regulating kinases allows MDM2 to interact with p53 and mediate proteasomal degradation [26]. WIP1 inactivation or depletion in tumor cells with high expression of WIP1 results in reduced cell viability in vitro or suppressed tumor growth in preclinical rodent models [6,27-31]. This is supported by the demonstration that Ppm1d-deficient mice show a delayed onset of mammary gland tumor development whereas an accelerated onset of tumors is evident in mice overexpressing PPM1D and ERBB2 in the mammary gland as well as reduced survival of $A P C^{\mathrm{min}}$ mice developing colon cancer $[27,32,33]$. Taken together, this suggests that WIP1 has important functions during tumorigenesis and that PPM1D has properties of an oncogene although no direct evidence for its being an oncogenic driver has been reported so far.

In an attempt to investigate the potential role of WIP1 during tumorigenesis we developed a transgenic mouse model overexpressing PPM1D. We show that this overexpression results in the development of cancers phenotypically similar to tumors arising in mice with dysfunctional p53 when subjected to low-dose irradiation.

\section{Results}

\subsection{Mice Carrying the PPM1D Transgene Develop Tumors in Response to Cellular Stress}

To characterize the role of PPM1D in tumor development, we constructed genetically engineered C57BL/6N mice overexpressing WIP1 through pronuclear injection of the human PPM1D gene controlled by the rat tyrosine hydroxylase $(\mathrm{TH})$ promoter (Figure 1A). Three founder lines were generated with intact transmission of the PPM1D transgene to following generations.

Elevated WIP1 protein expression was detected in the spleen, ovary, and intestine in mice hemizygous for PPM1D compared to wild-type mice (Figure 1B, File S1). However, no increase in tumor development was observed in these PPM1D-transgenic mice. We therefore backcrossed the C57BL/6N PPM1D +/ - mice for eight generations with $129 \times 1 / \mathrm{SvJ}$ mice, which are more prone to tumor formation, in order to establish a near $100 \% 129 \times 1 /$ SvJ background. In addition, given the wide-ranging actions of WIP1 during DNA damage repair, we hypothesized that overexpression of WIP under cellular stress could have the potential to induce neoplasms arising in response to extrinsic cellular DNA damage. Hence, PPM1D-overexpressing animals and their wild-type littermates were exposed to 4.5 Gy of sub-lethal whole-body irradiation at different ages (1-314 days old). 
Irradiated transgenic mice carrying the PPM1D gene had significantly higher tumor incidence ( $34 \%$ tumor probability) compared to wild-type mice $(7.8 \% ; p<0.0001) 100-300$ days post-irradiation (Figure 2A).

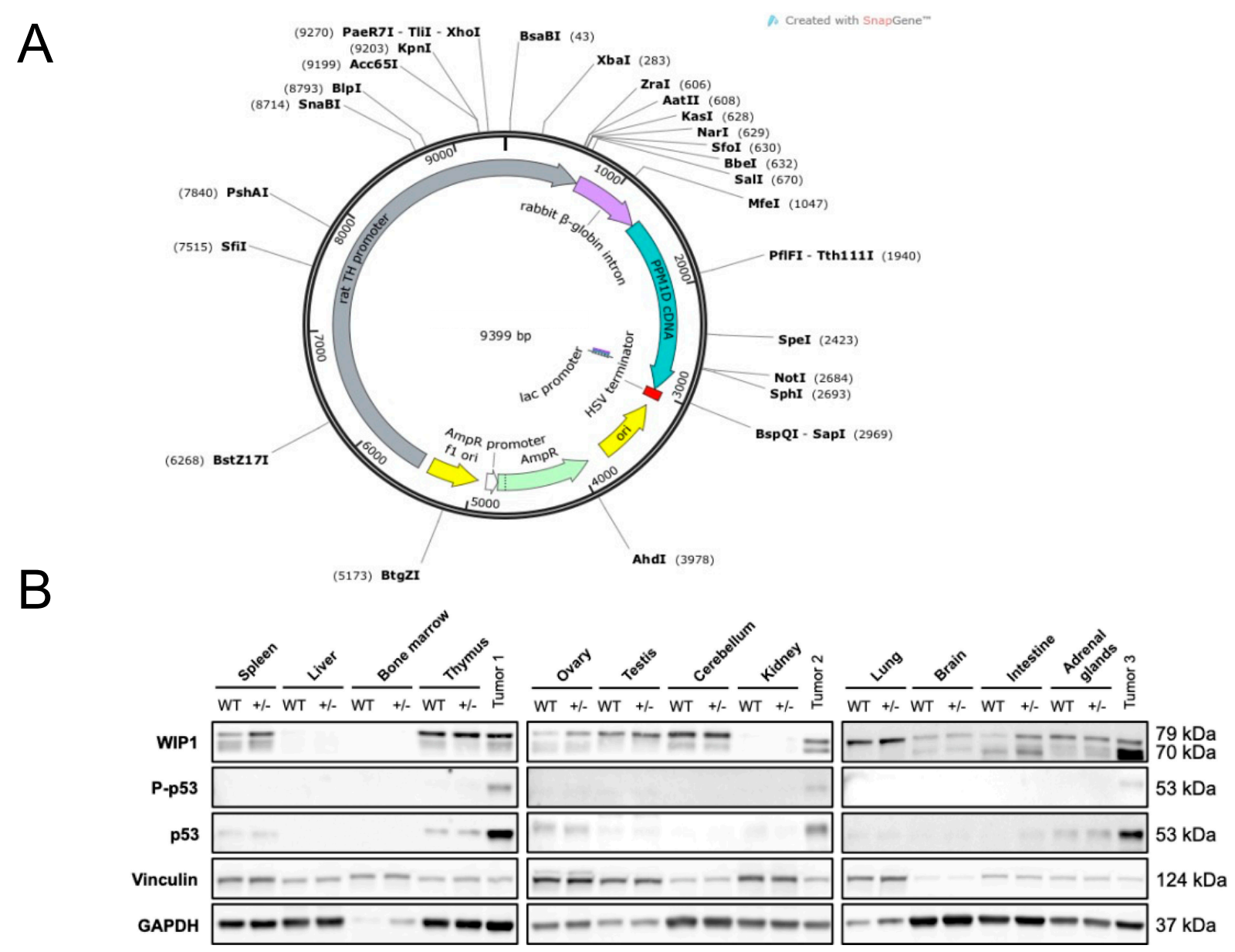

Figure 1. PPM1D-transgenic mice show increased WIP1 expression and develop widespread tumors with wild-type p53 accumulation. (A) Transgene construct. Human PPM1D cDNA (blue) PPM1D, ligated downstream of the rat tyrosine hydroxylase promoter (gray) and rabbit $\beta$-globin enhancer (violet). Herpes simplex virus (HSV) thymidine kinase gene sequence was used as a transcription terminator (red). (B) Thymic lymphoma shows increased WIP1 protein expression, p53 ${ }^{\text {Ser15 }}$ phosphorylation, and accumulation of total p53. Protein expression of WIP1 was analyzed in wild-type and PPM1D heterozygous $(+/-)$ mouse tissues. Three different mouse thymic lymphoma tumor samples were also included (tumors 1-3). Vinculin and GAPDH protein levels were used as loading controls.

The majority of cancers detected in PPM1D-transgenic mice were similar to those reported in irradiated p53-mutant mice post-irradiation [32-34], indicating a similar tumor phenotype caused by p53 impairment rather than p53 absence. Thymic lymphoblastic lymphoma was the most frequent malignancy observed ( $n=41$; Figure $2 B, C$ and Table 1$)$, compared to four thymic lymphomas detected in irradiated wild-type mice $(p<0.001)$. Other malignancies manifested in PPM1D-transgenic mice were leukemias/lymphomas $(n=9)$, different adenocarcinomas/adenomas $(n=18)$, and sarcomas $(n=4)$ (Table 1$)$. Additionally, we also identified mice with multiple primary tumors, one mouse with an adenocarcinoma in the lacrimal gland and an osteosarcoma with lung metastasis, one mouse with T-cell lymphoma, ovary carcinoma, and lung adenocarcinoma, two mice with neuroblastoma, one of which also had liver metastasis (Figure 2D), and a mouse with a gastric adenocarcinoma and leukemia (Table 1). Other tumor manifestations observed in several PPM1D-positive mice were spleen, liver, lung, and lymph node metastases 
(Figure 2E and Table 1). The odds ratio of developing cancer in mice with the PPM1D transgene was 6.3 (95\% confidence interval 2.7-14.2) compared to wild-type mice.

A

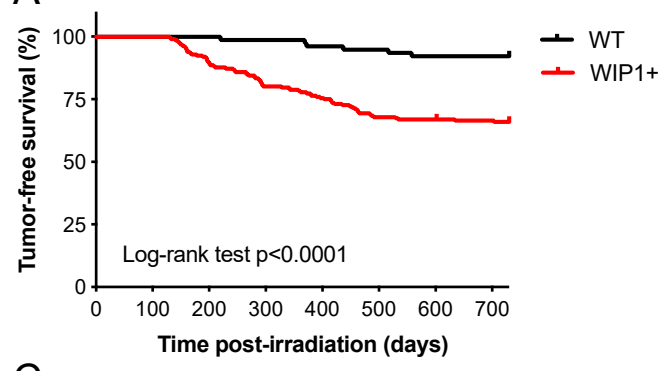

C

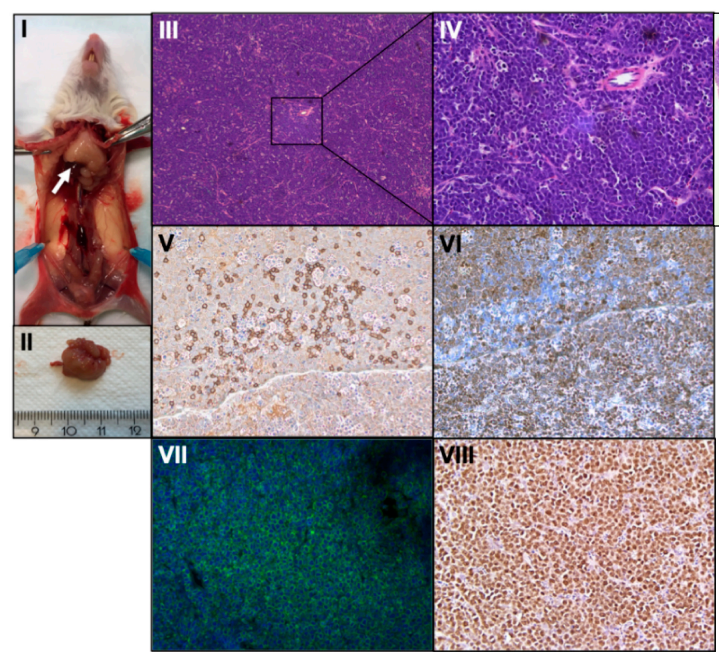

$\mathrm{E}$

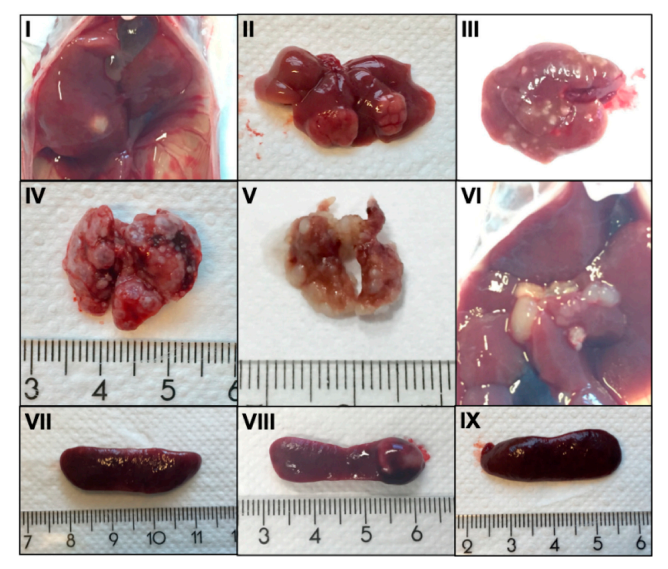

B

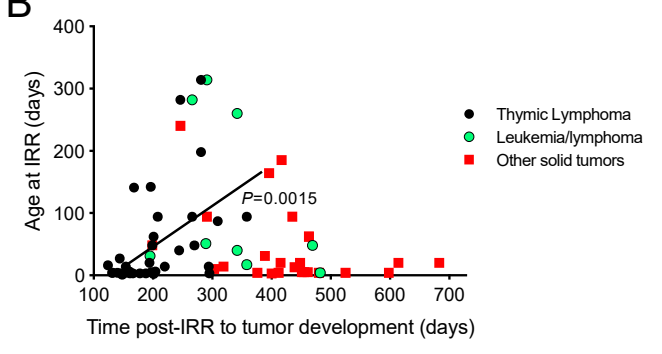

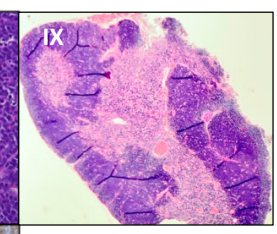

$\mathrm{D}$

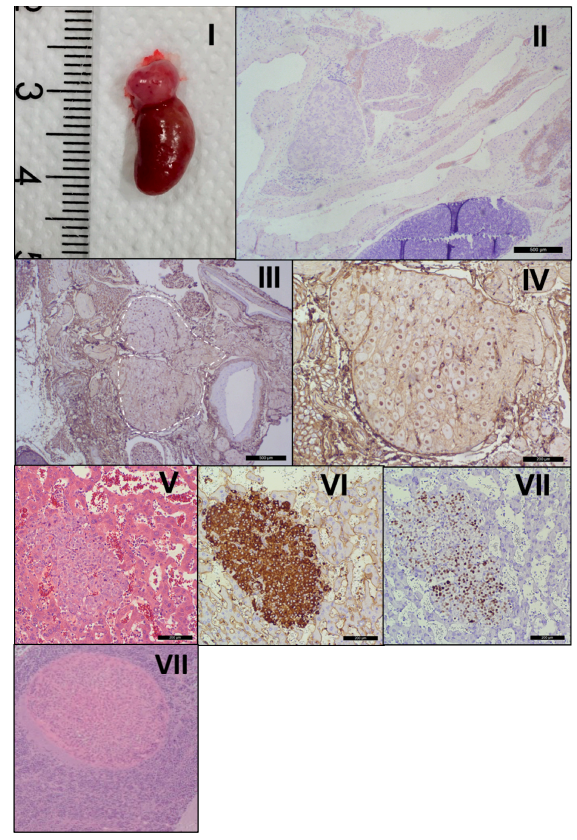

Figure 2. PPM1D/WIP1-transgenic mice have increased carcinogenic susceptibility compared to wild-type mice following irradiation. (A) Kaplan-Meier analysis of irradiated PPM1D/WIP1-transgenic mice with the endpoint defined as detection of tumors. Mice were either homozygous or heterozygous for the human PPM1D gene. WIP1-positive transgenic mice $(\mathrm{n}=210)$ and their wild-type littermates $(n=79)$ with different degrees of $129 \times 1 /$ SvJ-strain background were subjected to one 4.5 Gy sublethal whole-body irradiation dose at different days of age (1-314 days old). Mice that were positive for the human PPM1D gene frequently developed more tumors compared to wild-type mice (Log rank test, $p<0.0001$ ) following irradiation. Among the PPM1D/WIP1-positive mice, 69 mice developed tumors compared with six mice in the wild-type group (Fisher's exact test, $p<0.0001$ ). The odds ratio of developing cancer in the PPM1D/WIP1-positive group compared to the wild-type group was 6.3 (95\% confidence interval 2.7-14.2). (B) Time to tumor development of thymic lymphomas post-irradiation (IRR) correlated positively with age at irradiation (Pearson, $p=0.0015$. Spearman, $p=0.001$ ); the majority of lymphomas manifested $<300$ days. There was no correlation between age at irradiation and time to tumor development in 
mice diagnosed with other solid tumors (Pearson, $p=0.0658$, Spearman, $p=0.1589$ ) and mice diagnosed having leukemia/lymphoma (Pearson, $p=0.3651$, Spearman, $p=0.1977$ ). (C) Thymic lymphomas were the most common disease manifestation in irradiated human PPM1D/WIP1-positive mice. Representative image of thoracic tumor in situ indicated by the arrow (I) and after dissection (II). Microscopy at $4 \times(\mathrm{III})$ and $20 \times(\mathrm{IV})$ magnification after hematoxylin and eosin (H\&E) staining showed atypical lymphoblastic cells having intense mitotic activity. (V) Infiltration of B220-positive cells $(20 \times$ magnification). (VI-VII) The majority of cells are positive for the pan-T cell marker CD3 (20×magnification). (VIII) Staining for Ki-67 was highly positive, indicating high proliferative activity ( $20 \times$ magnification). The thoracic organs, mediastinum (heart base and hilum of the lung), thymus tumor, and lymph nodes displayed extensive infiltrates of neoplastic and highly malignant lymphoblasts consistent with lymphoblastic lymphoma. (IX) H\&E staining of a normal mouse thymus (4× magnification). (D) Adrenal neuroblastic tumor and liver metastasis from a PPM1D/WIP1 transgenic mouse. (I) Macroscopic view of the adrenal tumor. (II) Hematoxylin/eosin staining of the adrenal tumor. (III-IV) PHOX2B-staining was positive, indicating neural crest origin of this neuroblastoma-like tumor. (V-VII) Liver metastasis with hematoxylin/eosin staining, synaptophysin staining, and PHOX2B staining, respectively. (VII) H\&E staining of a normal mouse adrenal showing both the medulla and the cortex $(4 \times$ magnification). (E) PPM1D-transgenic mice exhibit widespread tumor malignancies. Representative macroscopic photographs: tumor mass in the liver (I), multiple hepatic metastases (II,III), lung carcinosis/metastases (IV,V), enlarged lymph nodes (VI), and splenomegaly (VII-IX) indicative of high tumor burden.

Table 1. List of tumors developed in irradiated PPM1D-transgenic mice, related to Figure 2.

\begin{tabular}{|c|c|c|c|c|}
\hline \multirow{2}{*}{ Diagnosis } & \multirow{2}{*}{$\mathbf{n}$} & Age at $\operatorname{Irr}^{b}$ & \multirow{2}{*}{$\begin{array}{l}\text { Time from Irr to Tumor } \\
\text { Development }^{b} \text { (Days) }\end{array}$} & \multirow{2}{*}{$\frac{\text { Metastatic }}{\text { Spread }^{c}}$} \\
\hline & & (Days) & & \\
\hline \multicolumn{5}{|c|}{$P P M 1 D$-positive mice } \\
\hline Thymic lymphoblastic lymphoma & 41 & Mdn: $6(1-314)$ & Mdn: 196 (124-535) & Systemic \\
\hline Leukemia/lymphoma & 9 & Mdn: 48 (4-314) & Mdn: 342 (195-482) & Systemic \\
\hline Other solid tumors & 22 & Mdn: 20 (3-240) & Mdn: 435 (246-683) & \\
\hline Papillary serous ovarian cancer & 5 & Mdn: 20 (4-164) & Mdn: 415 (389-598) & Splenomegaly \\
\hline Lacrimal gland tumor & 6 & Mdn: 455 (291-614) & Mdn: 20 (4-94) & Splenomegaly \\
\hline Gastrointestinal stromal tumor & 1 & 62 & 463 & Splenomegaly \\
\hline Gastric adenocarcinoma & 1 & 62 & 463 & Lung, splenomegaly \\
\hline Adenocarcinoma of the lung & 4 & Mdn: 414 (246-463) & Mdn: 34 (4-240) & \\
\hline Angiosarcoma: & 2 & & & \\
\hline$\# 1$ & & 48 & 199 & \\
\hline$\# 2$ & & 14 & 319 & Splenomegaly \\
\hline Osteosarcoma metastasis in the lung ${ }^{a}$ & 1 & 10 & 303 & Lung, Splenomegaly \\
\hline Neuroblastoma/Adrenal tumor: & 2 & & & \\
\hline$\# 1$ & & 6 & 683 & Liver, splenomegaly \\
\hline$\# 2$ & & 6 & 522 & \\
\hline \multirow[t]{2}{*}{ Total number of tumors } & 72 & & & \\
\hline & & Wild-type mice & & \\
\hline Thymic lymphoblastic lymphoma & 4 & Mdn: $16(14-87)$ & Mdn: 334 (220-596) & Systemic \\
\hline Adenocarcinoma of the lung & 1 & 14 & 437 & \\
\hline Lacrimal gland tumor & 1 & 17 & 517 & \\
\hline Total number of tumors & 6 & & & \\
\hline
\end{tabular}

${ }^{a}$ Presence of proliferative lymphoblastic cells in the spleen indicative of leukemia or lymphoma. ${ }^{b}$ Age in days given as median (Mdn) and range for groups. Irr; irradiation. ${ }^{c}$ And/or other tumor manifestations such as splenomegaly. Systemic spread implicate infiltrates of atypical lymphocytes/lymphoblasts engaging the spleen, liver, and in some cases, the kidneys. In total, 72 tumors were diagnosed in 69 PPM1D-positive mice (three mice were diagnosed as having two primary tumors each).

Comparison of age at irradiation and time to tumor development disclosed a positive correlation, showing that younger PPM1D-positive animals were more susceptible to developing thymic lymphomas in response to DNA damage $(p<0.01)$, the majority (38 out of 41 ) of which arose $<365$ days post-irradiation (Figure 2B). Protein expression of thymic lymphomas showed high WIP1 expression and increased phosphorylation of p53 compared to non-irradiated thymus from PPM1D-positive and wild-type mice (Figure 1B). 


\subsection{PPM1D-Derived Mouse Lymphoma Tumors Show Frequent Pten and Notch1 Mutations and} Activation of Notch Signaling

To further characterize the tumors that developed in PPM1D-transgenic mice, we performed whole-exome sequencing on PPM1D-derived T-cell lymphomas. We detected an average of 18 (range 9-34) somatic protein-changing mutations compared to non-irradiated wild-type control mice. Thymic tissues from the two control groups, irradiated wild-type mice, and irradiated PPM1D-positive mice without tumors, showed an average of two (range 1-4) and three (range 1-5) somatic protein-changing mutation variants, respectively, similar to nonirradiated non-tumorigenic wild-type mice (Table S1). These data indicate that irradiation alone was not responsible for the accumulation of DNA mutations observed in the tumors. Among the thymic lymphomas, we found Pten and Notch1 to be recurrently altered (Figure 3). In total, 15 activating Notch 1 mutations were detected in 9 of 15 lymphomas (60\%) and among these, five tumors had more than one mutation of the Notch1 gene. Similar to human cancers, the Notch1 missense variants were found to cluster in the heterodimerization domain (HD) while frameshift or nonsense variants were clustered to the proline-glutamic acid-serine-threonine (PEST) domain (Figure 3A). Of the 15 sequenced lymphomas, Pten aberrations were detected in 12 cases $(80 \%)$ consisting of homozygous deletions (nine cases), missense variants (two cases), and frameshift insertion (one case) (Figure 3B). The three missense and frameshift variants all showed a high level variant allele fraction (variant allele fraction $85-97 \%$ ) suggesting a near homozygous presence. Mutations of Pten and Notch1 were co-occurring in seven cases (46\%) (Table S1). In addition to Notch1 and Pten mutations, aberrations in genes previously associated to human cancers were also observed in Ikzf1, Kras, Rac2, Trp53, Ctnnb1, Gli3, Zfp3612, Mpdz, Dd3x, Foxp1, Pin1, and Ezh2 (Table S1).

A

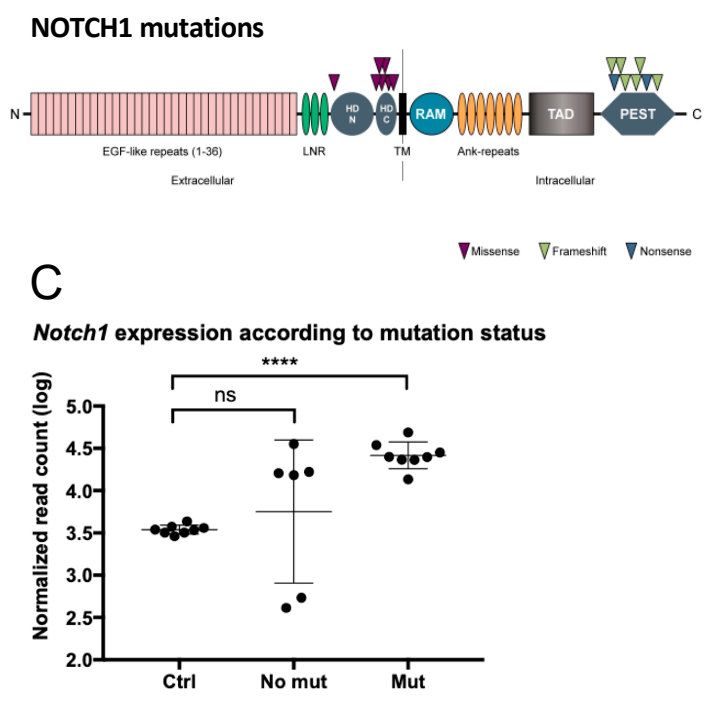

E

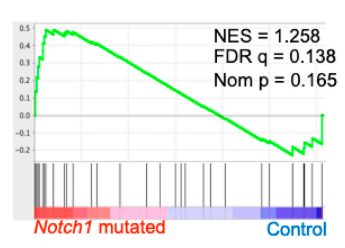

B

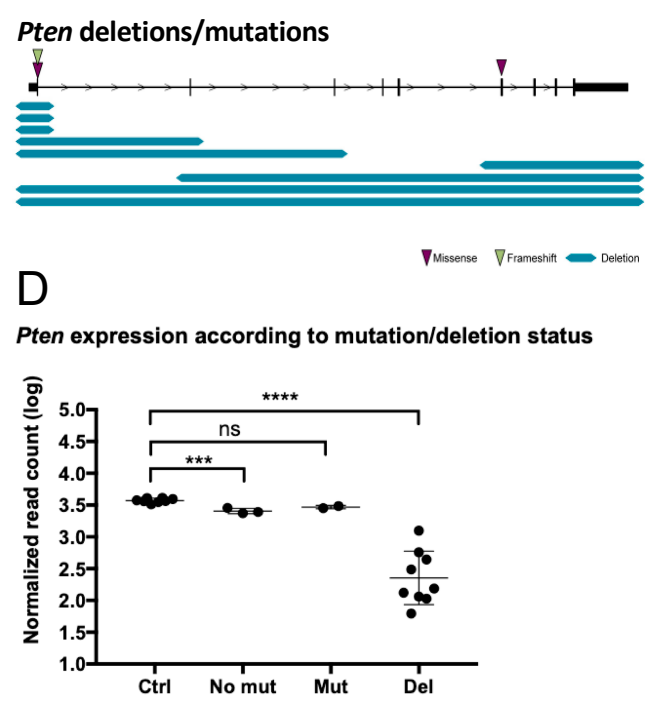

F

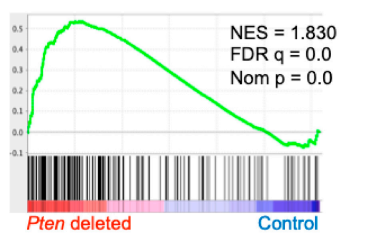

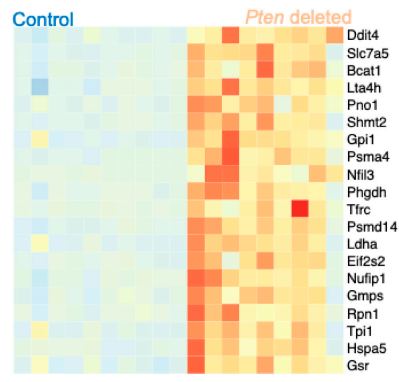

Figure 3. PPM1D-induced thymic lymphomas show typical Notch1 and Pten aberrations. (A) Schematic overview of the NOTCH1 protein showing the distribution of mutations in 15 whole-exome sequenced lymphomas. Purple triangles indicate 
missense mutations in the intracellular heterodimerization (HD) domain, whereas green and blue triangles indicate frameshift or nonsense mutations in the extracellular PEST domain. LNR, Lin/NOTCH repeats; HD, heterodimerization domain; TM, transmembrane domain; RAM, RBP-JK-associated molecule region; TAD, transactivation domain; PEST, sequence rich in proline, glutamic acid, serine, and threonine. (B) Diagram of the Pten gene showing the size and distribution of Pten deletions/mutations in lymphomas. The locations of the point mutations are shown with purple and green triangles above the gene diagram. The blue lines below the gene diagram show the size of the deletions identified by exome sequencing. (C) Expression of the Notch1 transcript in controls and tumors with and without Notch1 mutation. The y axis shows the log value of the number of RNA-seq reads mapping to the Notch1 gene. The difference between controls and tumors without Notch1 mutations is not significant (ns), whereas Notch1 expression is significantly higher in the mutated tumors (adjusted $p$ value $<0.0001$; Bonferroni multiple comparisons test). ${ }^{* * *} p<0.0001$. (D) Expression of Pten in controls and tumors with and without Pten mutation/deletion. Expression is significantly lower in tumors harboring deletions than in controls (adjusted $p$ value 0.0001 , Bonferroni multiple comparisons test). ${ }^{* * *} p<0.001,{ }^{* * * *} p<0.0001$. (E) Gene set enrichment analysis comparing Notch1 mutated tumors to controls demonstrated a significant upregulation of genes related to Notch signaling (MSigDB "Hallmarks" gene set). Left panel: Enrichment plot from GSEA. Right panel: Heatmap showing the expression (z scores) of core enriched genes. Red color indicates positive z scores; blue color indicates negative z scores. (F) Gene set enrichment analysis comparing Pten deleted tumors to controls demonstrated upregulation of genes related to the Mtorc1 pathway (MSigDB “Hallmarks" gene set). Left panel: Enrichment plot from GSEA. Right panel: Heatmap showing the expression (z scores) of the top 20 core enriched genes. Red color indicates positive z scores; blue color indicates negative $\mathrm{z}$ scores.

We also performed RNA-sequencing on the tumors and analyzed them using principal component analysis (PCA) and unsupervised hierarchical clustering based on the 200 most variable genes (Figure S1A,B). Differential gene expression analysis (Table S2) identified 4138 upregulated and 4378 downregulated genes in tumors when compared to controls. Tumors harboring Notch1 mutations had significantly higher expression of the Notch1 gene compared to controls (Figure 3C). Also, as expected, Pten expression was significantly lower in tumors harboring deletions of the gene (Figure 3D). Likewise, gene set enrichment analysis (GSEA) showed corresponding increased expression of genes involved in Notch- and Mtorc1-signalling in Notch1- or Pten-mutated tumors compared to controls (Figure 3E,F). Thus, tumor development was phenotypically and genetically similar to tumors from p53-impaired mice exhibiting Pten deletions and wild-type p53-accumulation [35-37] and to human thymic lymphomas which frequently harbor NOTCH mutations [35].

\section{Discussion}

Aberrant expression of PPM1D caused by chromosomal gains, gene amplification, or activating mutations has been described in multiple cancers and high expression of PPM1D often correlates with poor patient prognosis [38]. Moreover, high expression and/or genetic aberration of PPM1D is frequently found in cancers with wtTRP53 suggesting that high protein levels or stability of WIP1 inhibit the activity of p53 that can results in neoplastic transformation and malignant tumor formation [36,37]. For these reasons, multiple inhibitors targeting the anti-tumorigenic activity of PPM1D have been developed [39]. Although some of these inhibitors have proven to be effective in inhibiting tumor cell growth both in vitro and in vivo, none of the inhibitors have been subjected to clinical trial due to poor solubility or substandard pharmacokinetics [39]. The demonstration that Ppm1d-deficient mice significantly delayed the formation of ERBB2-induced mammary tumors and that Ppm1d-null embryonic mouse fibroblasts were resistant to transformation by RAS, ERBB2, and c-MYC [27] encouraged us to establish a genetically modified mouse model overexpressing WIP1. PPM1D-transgenic mice developed tumors of different origin after low-dose irradiation compared to wild-type control mice. The spectrum of observed tumors was highly similar to tumors observed in p53-deficient mice or p53-deficient mice subjected to irradiation [32-34,40]. Interestingly, the tumors which developed in our PPM1D-transgenic mice are phenotypical similar to mice with Trp53 mutations but not similar to Trp53 knockout mice. Mice with Trp53 deletions mainly develop lymphomas and less frequently sarcomas $[33,40]$ whereas Trp53 mutant mice, in 
addition to lymphomas and more frequently sarcoma, also develop carcinomas [34]. This is similar to the spectrum of tumors observed in our PPM1D-transgenic mice. Thymic lymphoma derived from PPM1D-transgenic mice expresses high levels of wild-type p53 and WIP1 similar to Trp53 mutated mice showing high levels of mutated p53 [41]. Several human cancers exhibit PPM1D gene amplification and/or overexpression of the WIP1 protein. The majority of these tumors also retain wild-type p53 but the functionality is compromised $[8,14]$. We subjected a group of our mice to low-dose irradiation that induces cellular stress and DNA damage and activates p53. Activation of $\mathrm{p} 53$ promotes enhanced expression of endogenous PPM1D and WIP1 will dephosphorylate serine and threonine residues on key proteins involved in the DNA damage response, including pSer15 on p53. Increased expression of WIP1 will also stabilize MDM2 and MDMX by dephosphorylation of pSER395 and pSer403, respectively [39]. Enhanced MDM2/MDMX stability target p53 for MDM2-mediated ubiquitination and proteasomal degradation. Together this suggests that high levels of WIP1 protein compromise p53 activity in these cancers.

In addition, both the genomic landscape and expression profiles obtained from DNA and RNA sequencing of PPM1D-induced tumors had similar features with regard to DNA mutations and expression profiles as observed in tumors deriving from p53-impaired mice [42]. The majority of sequenced thymic lymphomas detected in PPM1D-transgenic mice contained deletions in the tumor-suppressor gene Pten. In $\mathrm{p}^{-/-}$mice developing thymic lymphomas, deletion of Pten is an early event occurring in a stem or early T-cell lineage progenitor before rearrangements of the T-cell receptors [42]. We also detected frequent activating Notch1 mutations in lymphomas from PPM1D-transgenic mice. Activating NOTCH1 mutations is one of the most frequent gene aberration found in human T-cell acute leukemia/lymphoma and the majority of these mutations are located in the PEST or heterodimerization domain [35] similar to the Notch1 mutations detected in lymphomas from PPM1D-transgenic mice. In contrast, lymphomas from $\mathrm{p}^{53^{-/-}}$mice do not exhibit Notch1 mutation but instead frequently contain activating mutation of Ikaros resulting in constitutive activation of the Notch signaling pathway [42].

Other studies have shown that overexpression of PPM1D together with oncogenes augmented tumor formation in mice or resulted in reduced the survival of mice deficient of the APC tumor-suppressor gene [3,43]. Our results show that PPM1D overexpression results in tumor development in mice subjected to DNA stresses. Together this strongly suggests that PPM1D is an oncogenic driver through its ability to repress p53 activity that leads to deviant cell cycle arrest, DNA repair, apoptosis, and tumor development.

\section{Materials and Methods}

\subsection{Ethical Permits}

The animal experiments were approved by the regional ethics committee for animal research in Northern Stockholm, appointed and under the control of the Swedish Board of Agriculture and the Swedish Court. All animal experiments were in accordance with national regulations (SFS 1988:534, SFS 1988:539, and SFS 1988:541). For specific approval numbers, please refer to the sections below.

\subsection{WIP1-Overexpressing Mouse Model}

Transgenic WIP1-overexpressing mice were generated by pronuclear injection with random integration of a PPM1D-transgenic vector construct, carried out at Karolinska Center for Transgene Technologies (KCTT) and granted according to ethical approval numbers N251-12 and N42-14.

The construct consisted of rat tyrosine hydroxylase $(\mathrm{TH})$ promoter, the rabbit betaglobin intron to enhance expression, and cDNA for the human PPM1D gene (MGC Human PPM1D Sequence-Verified cDNA, Clone Id: 5167004, Dharmacon). Herpes simplex virus (HSV) thymidine kinase gene sequence was used as a transcription terminator (Figure 1A). The plasmid was kindly provided by Prof. William Weiss (University of California, San Francisco, CA, USA). 
The linearized and purified construct was diluted to $1.5 \mathrm{ng} / \mathrm{ul}$ in microinjection buffer (10 mM Tris- $\mathrm{HCl}, \mathrm{pH7} .4,0.1 \mathrm{mM}$ EDTA) and injected into the pronucleus of C57BL/6NCrl zygotes using a Nikon TE200 microinjection system with Narishige NT-88NEN micromanipulators and a Warner Instruments PLI-100A pico-liter injector. The microinjected embryos were transferred into the oviducts of pseudopregnant Crl:CD1(ICR) female mice using standard surgery techniques and ear biopsies from the resulting 55 offspring were screened for the presence of the PPM1D transgene by PCR using forward primer: $5^{\prime}$ CTGGTCATCATCCTGCCTTTCT- ${ }^{\prime}$ and reverse primer: $5^{\prime}$-GCCTTTCCCCGAGACTTCG$3^{\prime}$ (Sigma-Aldrich, St. Louis, MO, USA). Six transgenic animals were found of which four founder animals were further established based on their ability to pass on the human PPM1D-transgene to their offspring.

In order to achieve a more tumor-permissive genetic background, these four transgenic mouse-lines were backcrossed with 129X1/SvJ mice, aiming for ten generations of backcrossing which should result in approximately 100\% 129X1/SvJ background. Backcrossing was carried out in accordance with ethical permit number N641-12. Throughout breeding, mice were monitored closely for development of palpable abdominal tumors or other disease manifestations up to 548 days (1.5 years) of age.

\subsection{Irradiation of Mice}

Using a linear accelerator (X-RAD 320, Biological Irradiator, North Branford, CT, USA) with a dose rate of $0.95 \mathrm{~Gy} / \mathrm{min}$ at $320 \mathrm{KV}$ and a radiation field of $20 \times 20 \mathrm{~cm}$, PPM1Dtransgenic mice from three established transgenic lines and their wild-type littermates resulting from heterozygous breeding pairs with different degrees of 129X1/SvJ strain background (three to seven generations of backcrossing from C57BL/6N to 129X1/SvJ background) were subjected to whole-body irradiation at a single sublethal dose of $4.5 \mathrm{~Gy}$ (ethical approval N290-15), after which they were monitored daily in their usual pathogenfree environment. Mice were exposed to irradiation at different ages (1 to 314 days old). Littermates were always irradiated simultaneously. The mice were followed up to 548 days (1.5 years) of age.

\subsection{Immunoblotting}

Protein extraction, determination of protein content, SDS-PAGE under reducing conditions, electroblot, and immunoreaction detection were carried out as previously described [44]. Briefly, total proteins were extracted using RIPA buffer (Thermo Fisher Scientific, Waltham, MA, USA) supplemented with Halt ${ }^{\mathrm{TM}}$ Protease and Phosphatase Inhibitor Cocktail (Thermo Fisher Scientific) and 50 ug of total protein were resolved by reducing SDS-PAGE and transferred onto a nitrocellulose membrane. The membranes were blocked with 5\% non-fat milk in TBS-T bufferand incubated with anti-WIP1, anti-phospho-p53, and anti-p53, all from Cell Signaling, diluted 1:1000 at $4{ }^{\circ} \mathrm{C}$ overnight. Anti-Vinculin and antiGADPH from Abcam were diluted 1:4000 and 1:10,000, respectively, at room temperature for $1 \mathrm{~h}$. Chemiluminescence visualization of antibodies was performed with Amersham ${ }^{\mathrm{TM}}$ ECL $^{\mathrm{TM}}$ Prime Western Blotting Detection Reagent (GE Healthcare). Visualization and imaging of signal was performed with ImageQuant LAS 4000 (GE Healthcare).

\subsection{Immunohistochemistry of Transgenic Mouse Tissue Samples}

Formalin-fixed and paraffin-embedded transgenic mouse tissue sections were deparaffinized in xylene and graded alcohols, hydrated, and washed in phosphate-buffered saline (PBS). After antigen retrieval in sodium citrate buffer (pH 6) in a microwave oven, endogenous peroxidase activity was blocked by $0.3 \% \mathrm{H}_{2} \mathrm{O}_{2}$ for $15 \mathrm{~min}$. Biotin blocking was preformed using an avidin/biotin blocking kit (Vector Laboratories). All washes and dilutions were performed in PBS containing $0.1 \%$ saponin. Sections were incubated with an anti-Ki-67 antibody (clone SP6, ab16668, abcam), anti-B220 antibody (clone RA3-6B2, R\&D Systems, Minneapolis, MN, USA), or anti-CD3 antibody (SP7, Abcam, Cambridge, UK) containing 3\% human serum overnight at room temperature. After blocking with 
$1 \%$ goat serum for 15 min sections were incubated with a biotin-conjugated secondary antibody (goat anti-rabbit IgG or goat anti rat IgG, Vector laboratories) containing $1 \%$ goat and 3\% human serum at room temperature for $30 \mathrm{~min}$. For detection of an $\mathrm{ABC}$ complex (Elite $\mathrm{ABC}$ kit, Vector laboratories) was used before the sections were developed using diaminobenzidine (DAB Peroxidase Substrate kit; Vector Laboratories) as chromogen. Sections were counterstained with Mayer's hematoxylin (Histolab). For immunofluorescence detection of CD3-positive cells, sections were incubated with anti-CD3 antibody (SP7, Abcam, Cambridge, UK) in $4 \%$ goat serum overnight at $4{ }^{\circ} \mathrm{C}$ following incubation with secondary Goat anti-rabbit-Alexa Fluor 488 (Thermo fisher Scientific). Histological assessment of the transgenic mouse tissues was performed by a pathologist. For full list of antibodies used for immunohistological analyses see Table S3.

\subsection{Whole-Exome Sequencing, Variant Calling, and Copy Number Alterations in Mice}

Whole-exome sequencing (WES) was performed on DNA from totally 24 samples; 15 independently developed thymic lymphomas and thymus from nine controls. The controls were divided into three different groups corresponding to (a) irradiated PPM1D-transgenes, (b) irradiated wild-type, and (c) non-irradiated wild-type. In the respective control groups, three mice, representing three different founder strains, were subjects for sequencing.

WES was performed by Otogenetics (Otogenetics Corporation, Atlanta, GA, USA) through pair-end sequencing on Illumina platforms (Illumina, San Diego, CA, USA) after enrichment with Agilent SureSelectXT Mouse All Exon (Agilent technologies, Santa Clara, CA, USA) reaching an average coverage of 80X (range 47.5-99.2X) (Table S1). Read trimming, mapping to the mouse reference genome $\mathrm{mm} 10$, and variant calling were performed using CLC Genomics Workbench 5.0 software (CLC, Aahus, Denmark). Somatic calling of lymphoblastic tumors and irradiated controls was done using the combined sequence from the three non-irradiated wild-type mice as normal control. Only high quality called variants with a minimum $10 \%$ allele frequency and a total read coverage of ten were considered for further analysis. All synonymous variants or variants in non-coding regions except those affecting canonical splice sites were discarded. Remaining variants were assessed manually through the Integrative Genomics Viewer (https://software.broadinstitute.org/software/igv/ accessed on 8 September 2020) for removal of calls due to mapping artifacts or paralogs. Calling and visualization of copy number alterations was done using the software Control-Free (control-FREE Copy Number Caller) that generates rations from normalized read distribution between tumor and normal followed by visualization in a Shiny application as described previously [45].

\subsection{RNA Sequencing of Mouse Tumors}

Bulk RNA sequencing (RNA-seq) was performed on extracted RNA in a total of 24 samples; 10 controls (thymic tissue from healthy mice) and 14 thymic lymphomas. As with mouse tumor WES, controls were divided into four groups based on irradiation status and genotype: wild-type non-irradiated $(n=3), P P M 1 D$-transgenic non-irradiated $(n=3)$, wild-type irradiated $(n=2)$, and PPM1D-transgenic irradiated $(n=2)$. Total RNA extraction, library preparation, and sequencing were performed by Otogenetics (Otogenetics Corporation, Atlanta, GA, USA) through paired-end sequencing on Illumina HiSeq 2500 (Illumina, San Diego, CA, USA) after mRNA purification using the TruSeq Stranded cDNA kit. Read length was 100-125 bp for all samples.

Samples were aligned to the mm10 reference genome using STAR in 2-pass mode. Aligned reads were quantified using htseq-count and differential gene expression analysis was performed using the R/Bioconductor package DESeq2. After having converted mouse Ensembl gene IDs to human orthologs using the R package gOrth, gene set enrichment analysis was performed using the GSEA software from Broad institute and the Hallmark collection of gene sets from the Molecular Signatures Database, MSigDB, version 6.2. 


\subsection{Statistical Analysis}

Statistical analyses were done with GraphPad Prism software (GraphPad Software, San Diego, CA, USA). The $\mathrm{IC}_{50}$ values (inhibitory concentration 50\%) were determined from log concentrations-effect curves using non-linear regression analysis. $\mathrm{T}$ test was used to compare means between two groups and for comparison of three or more groups, one-way ANOVA followed by Bonferroni multiple-comparisons post-test were used. Survival analysis was examined with log-rank test and Fisher's test was used to test significance of association between the two categories. Correlations were assessed with Pearson test/Spearman non-parametric test. $p<0.05$ was considered significant and all tests were two-sided. Survival curves were calculated using the Kaplan-Meier method. Regarding statistical methods for genomics and transcriptomics studies, please refer to the corresponding methods section.

\section{Conclusions}

Taken together this study shows that PPM1D can act as an oncogenic driver inducing tumors similar to tumors induced by p53 impairments.

Supplementary Materials: The following are available online at https:/ /www.mdpi.com/article/10 .3390 /cancers13215493/s1, Figure S1: PPM1D/WIP1-transgenic mice demonstrate clear distinction between thymic lymphoma tissue and control tissue, Table S1: Genomic alterations detected in PPM1D-transgenic mouse tumors through WES, Table S2: Differential gene expression analysis on PPM1D-transgenic mouse tumors, Table S3: List of antibodies used in transgenic mouse tissue analysis by Western blot and immunohistochemistry. File S1: Original Western blot images of transgenic-PPM1D murine tissue corresponding to Figure 1B.

Author Contributions: Conceptualization, J.M., J.I.J. and P.K.; methodology, J.M., S.F., M.G., T.K.O., G.G.-O. and M.W.; software, J.M., S.F., T.K.O. and G.G.-O.; validation, J.M., S.F., M.G., T.K.O., D.T., G.G.-O., L.H.M.E. and M.W.; formal analysis, J.M., S.F., M.G., T.K.O., D.T., G.G.-O., M.W., T.M., J.I.J. and P.K.; investigation, J.M., S.F., M.G., T.K.O., D.T., G.G.-O., L.H.M.E. and M.W.; resources, T.M., J.I.J. and P.K.; data curation, J.M., S.F., M.G., T.K.O., G.G.-O. and M.W.; writing-original draft preparation, J.M., N.B., J.I.J. and P.K.; writing-review and editing, J.M., S.F., D.T., N.B., J.I.J. and P.K.; visualization, J.M., S.F., M.G., T.K.O., G.G.-O. and M.W.; supervision, J.I.J. and P.K.; project administration, J.M., J.I.J. and P.K.; funding acquisition, J.I.J. and P.K. All authors have read and agreed to the published version of the manuscript.

Funding: This research was funded in part by the Swedish Childhood Cancer Foundation, the Swedish Research Council, the Swedish Cancer Foundation, the Swedish Foundation for Strategic Research, Karolinska Institutet, Märta and Gunnar V Philipson Foundation, and The Cancer Research Foundations of Radiumhemmet, and the APC was funded by the Swedish Cancer Foundation (Grant no: CAN2018/460, JI Johnsen).

Institutional Review Board Statement: The study was conducted according to the guidelines of the Declaration of Helsinki, and approved by the regional ethics committee for animal research in Northern Stockholm, appointed and under the control of the Swedish Board of Agriculture and the Swedish Court. All animal experiments were in accordance with national regulations (SFS 1988:534, SFS 1988:539, and SFS 1988:541). Ethical approval numbers: N251-12 and N42-14.

Informed Consent Statement: Not applicable.

Data Availability Statement: The WES data presented in this study are openly available in European Nucleotide Archive (ENA) study accession: http:/ / www.ebi.ac.uk/ena/data/view/PRJEB40261 accessed on 8 September 2020. RNA sequencing data from mouse thymic lymphomas are available at GEO (Gene Expression Omnibus) under the accession number GSE157769 (token: kjsfomymtluzpsl) accessed on 25 September 2020.

Acknowledgments: We wish to thank Karolinska Center for Transgene Technologies (KCTT) for the transgenic production service, WA. Weiss and L. Chesler for providing the construct vectors used for generating transgenic PPM1D-mice, and Clinical Genomics, SciLifeLab, Gothenburg, Sweden and the Bioinformatics Core Facility platforms at the Sahlgrenska Academy, University of Gothenburg, Gothenburg, Sweden for assistance with the bioinformatical analysis of sequencing data. We thank 
Teodora Andonova, Inger Bodin, and Susanne Ahlberg for their help and contribution to this work. Special thanks to Jessica Lundgren and Selameyhune Assefa for their excellent assistance with the PPM1D-transgenic animals. We convey our gratitude to S.J. Mazur and E. Appella for useful critical assessment of our manuscript. This work was supported with grants from the Swedish Childhood Cancer Foundation, the Swedish Research Council, the Swedish Cancer Foundation, the Swedish Foundation for Strategic Research, Karolinska Institutet, Märta and Gunnar V Philipson Foundation, and The Cancer Research Foundations of Radiumhemmet. The study sponsors had no role in the design of the study; the collection, analysis, and interpretation of the data; the writing of the manuscript; or the decision to submit the manuscript for publication.

Conflicts of Interest: The authors have no conflict of interest to declare.

\section{References}

1. Fiscella, M.; Zhang, H.; Fan, S.; Sakaguchi, K.; Shen, S.; Mercer, W.E.; Vande Woude, G.F.; O'Connor, P.M.; Appella, E. Wip1, a novel human protein phosphatase that is induced in response to ionizing radiation in a p53-dependent manner. Proc. Natl. Acad. Sci. USA 1997, 94, 6048-6053. [CrossRef]

2. Fujimoto, H.; Onishi, N.; Kato, N.; Takekawa, M.; Xu, X.Z.; Kosugi, A.; Kondo, T.; Imamura, M.; Oishi, I.; Yoda, A.; et al. Regulation of the antioncogenic Chk2 kinase by the oncogenic Wip1 phosphatase. Cell Death Differ. 2006, 13, 1170-1180. [CrossRef]

3. Lu, X.; Nannenga, B.; Donehower, L.A. PPM1D dephosphorylates Chk1 and p53 and abrogates cell cycle checkpoints. Genes Dev. 2005, 19, 1162-1174. [CrossRef]

4. Lu, X.; Nguyen, T.A.; Donehower, L.A. Reversal of the ATM/ATR-mediated DNA damage response by the oncogenic phosphatase PPM1D. Cell Cycle 2005, 4, 1060-1064. [CrossRef] [PubMed]

5. Oliva-Trastoy, M.; Berthonaud, V.; Chevalier, A.; Ducrot, C.; Marsolier-Kergoat, M.C.; Mann, C.; Leteurtre, F. The Wip1 phosphatase (PPM1D) antagonizes activation of the Chk2 tumour suppressor kinase. Oncogene 2007, 26, 1449-1458. [CrossRef] [PubMed]

6. Shreeram, S.; Hee, W.K.; Demidov, O.N.; Kek, C.; Yamaguchi, H.; Fornace, A.J., Jr.; Anderson, C.W.; Appella, E.; Bulavin, D.V. Regulation of ATM/p53-dependent suppression of myc-induced lymphomas by Wip1 phosphatase. J. Exp. Med. 2006, 203, 2793-2799. [CrossRef] [PubMed]

7. Yoda, A.; Xu, X.Z.; Onishi, N.; Toyoshima, K.; Fujimoto, H.; Kato, N.; Oishi, I.; Kondo, T.; Minami, Y. Intrinsic kinase activity and SQ/TQ domain of Chk2 kinase as well as N-terminal domain of Wip1 phosphatase are required for regulation of Chk2 by Wip1. J. Biol. Chem. 2006, 281, 24847-24862. [CrossRef] [PubMed]

8. Castellino, R.C.; De Bortoli, M.; Lu, X.; Moon, S.H.; Nguyen, T.A.; Shepard, M.A.; Rao, P.H.; Donehower, L.A.; Kim, J.Y. Medulloblastomas overexpress the p53-inactivating oncogene WIP1/PPM1D. J. Neurooncol. 2008, 86, 245-256. [CrossRef]

9. Fuku, T.; Semba, S.; Yutori, H.; Yokozaki, H. Increased wild-type p53-induced phosphatase 1 (Wip1 or PPM1D) expression correlated with downregulation of checkpoint kinase 2 in human gastric carcinoma. Pathol. Int. 2007, 57, 566-571. [CrossRef]

10. Hirasawa, A.; Saito-Ohara, F.; Inoue, J.; Aoki, D.; Susumu, N.; Yokoyama, T.; Nozawa, S.; Inazawa, J.; Imoto, I. Association of 17q21-q24 gain in ovarian clear cell adenocarcinomas with poor prognosis and identification of PPM1D and APPBP2 as likely amplification targets. Clin. Cancer Res. 2003, 9, 1995-2004.

11. Lambros, M.B.; Natrajan, R.; Geyer, F.C.; Lopez-Garcia, M.A.; Dedes, K.J.; Savage, K.; Lacroix-Triki, M.; Jones, R.L.; Lord, C.J.; Linardopoulos, S.; et al. PPM1D gene amplification and overexpression in breast cancer: A qRT-PCR and chromogenic in situ hybridization study. Mod. Pathol. 2010, 23, 1334-1345. [CrossRef] [PubMed]

12. Nikbakht, H.; Panditharatna, E.; Mikael, L.G.; Li, R.; Gayden, T.; Osmond, M.; Ho, C.Y.; Kambhampati, M.; Hwang, E.I.; Faury, D.; et al. Spatial and temporal homogeneity of driver mutations in diffuse intrinsic pontine glioma. Nat. Commun. 2016, 7, 11185. [CrossRef] [PubMed]

13. Ruark, E.; Snape, K.; Humburg, P.; Loveday, C.; Bajrami, I.; Brough, R.; Rodrigues, D.N.; Renwick, A.; Seal, S.; Ramsay, E.; et al. Mosaic PPM1D mutations are associated with predisposition to breast and ovarian cancer. Nature 2013, 493, 406-410. [CrossRef]

14. Saito-Ohara, F.; Imoto, I.; Inoue, J.; Hosoi, H.; Nakagawara, A.; Sugimoto, T.; Inazawa, J. PPM1D is a potential target for 17q gain in neuroblastoma. Cancer Res. 2003, 63, 1876-1883. [PubMed]

15. Tan, D.S.; Lambros, M.B.; Rayter, S.; Natrajan, R.; Vatcheva, R.; Gao, Q.; Marchio, C.; Geyer, F.C.; Savage, K.; Parry, S.; et al. PPM1D is a potential therapeutic target in ovarian clear cell carcinomas. Clin. Cancer Res. 2009, 15, 2269-2280. [CrossRef]

16. Xie, M.; Lu, C.; Wang, J.; McLellan, M.D.; Johnson, K.J.; Wendl, M.C.; McMichael, J.F.; Schmidt, H.K.; Yellapantula, V.; Miller, C.A.; et al. Age-related mutations associated with clonal hematopoietic expansion and malignancies. Nat. Med. 2014, 20, 1472-1478. [CrossRef] [PubMed]

17. Zhang, Y.; Sun, H.; He, G.; Liu, A.; Wang, F.; Wang, L. WIP1 regulates the proliferation and invasion of nasopharyngeal carcinoma in vitro. Tumour Biol. 2014, 35, 7651-7657. [CrossRef]

18. Bykov, V.J.N.; Eriksson, S.E.; Bianchi, J.; Wiman, K.G. Targeting mutant p53 for efficient cancer therapy. Nat. Rev. Cancer 2018, 18, 89-102. [CrossRef] [PubMed]

19. Martin, B.; Verdebout, J.M.; Mascaux, C.; Paesmans, M.; Rouas, G.; Verhest, A.; Ninane, V.; Sculier, J.P. Expression of p53 in preneoplastic and early neoplastic bronchial lesions. Oncol. Rep. 2002, 9, 223-229. [CrossRef] [PubMed] 
20. Bond, G.L.; Hu, W.; Levine, A.J. MDM2 is a central node in the p53 pathway: 12 years and counting. Curr. Cancer Drug Targets 2005, 5, 3-8. [CrossRef]

21. Michael, D.; Oren, M. The p53-Mdm2 module and the ubiquitin system. Semin. Cancer Biol. 2003, 13, 49-58. [CrossRef]

22. Canman, C.E.; Lim, D.S.; Cimprich, K.A.; Taya, Y.; Tamai, K.; Sakaguchi, K.; Appella, E.; Kastan, M.B.; Siliciano, J.D. Activation of the ATM kinase by ionizing radiation and phosphorylation of p53. Science 1998, 281, 1677-1679. [CrossRef]

23. Maya, R.; Balass, M.; Kim, S.T.; Shkedy, D.; Leal, J.F.; Shifman, O.; Moas, M.; Buschmann, T.; Ronai, Z.; Shiloh, Y.; et al. ATMdependent phosphorylation of Mdm2 on serine 395: Role in p53 activation by DNA damage. Genes Dev. 2001, 15, 1067-1077. [CrossRef]

24. Tibbetts, R.S.; Brumbaugh, K.M.; Williams, J.M.; Sarkaria, J.N.; Cliby, W.A.; Shieh, S.Y.; Taya, Y.; Prives, C.; Abraham, R.T. A role for ATR in the DNA damage-induced phosphorylation of p53. Genes Dev. 1999, 13, 152-157. [CrossRef] [PubMed]

25. Rossi, M.; Demidov, O.N.; Anderson, C.W.; Appella, E.; Mazur, S.J. Induction of PPM1D following DNA-damaging treatments through a conserved p53 response element coincides with a shift in the use of transcription initiation sites. Nucleic Acids Res. 2008, 36, 7168-7180. [CrossRef] [PubMed]

26. Lu, X.; Ma, O.; Nguyen, T.A.; Jones, S.N.; Oren, M.; Donehower, L.A. The Wip1 Phosphatase acts as a gatekeeper in the p53-Mdm2 autoregulatory loop. Cancer Cell 2007, 12, 342-354. [CrossRef] [PubMed]

27. Bulavin, D.V.; Phillips, C.; Nannenga, B.; Timofeev, O.; Donehower, L.A.; Anderson, C.W.; Appella, E.; Fornace, A.J., Jr. Inactivation of the Wip1 phosphatase inhibits mammary tumorigenesis through p38 MAPK-mediated activation of the p16(Ink4a)-p19(Arf) pathway. Nat. Genet. 2004, 36, 343-350. [CrossRef] [PubMed]

28. Demidov, O.N.; Timofeev, O.; Lwin, H.N.; Kek, C.; Appella, E.; Bulavin, D.V. Wip1 phosphatase regulates p53-dependent apoptosis of stem cells and tumorigenesis in the mouse intestine. Cell Stem Cell 2007, 1, 180-190. [CrossRef]

29. Doucette, T.A.; Yang, Y.; Pedone, C.; Kim, J.Y.; Dubuc, A.; Northcott, P.D.; Taylor, M.D.; Fults, D.W.; Rao, G. WIP1 enhances tumor formation in a sonic hedgehog-dependent model of medulloblastoma. Neurosurgery 2012, 70, 1003-1010. [CrossRef] [PubMed]

30. Nannenga, B.; Lu, X.; Dumble, M.; Van Maanen, M.; Nguyen, T.A.; Sutton, R.; Kumar, T.R.; Donehower, L.A. Augmented cancer resistance and DNA damage response phenotypes in PPM1D null mice. Mol. Carcinog. 2006, 45, 594-604. [CrossRef] [PubMed]

31. Wen, J.; Lee, J.; Malhotra, A.; Nahta, R.; Arnold, A.R.; Buss, M.C.; Brown, B.D.; Maier, C.; Kenney, A.M.; Remke, M.; et al. WIP1 modulates responsiveness to Sonic Hedgehog signaling in neuronal precursor cells and medulloblastoma. Oncogene 2016, 35, 5552-5564. [CrossRef] [PubMed]

32. Armstrong, J.F.; Kaufman, M.H.; Harrison, D.J.; Clarke, A.R. High-frequency developmental abnormalities in p53-deficient mice. Curr. Biol. 1995, 5, 931-936. [CrossRef]

33. Donehower, L.A.; Harvey, M.; Slagle, B.L.; McArthur, M.J.; Montgomery, C.A., Jr.; Butel, J.S.; Bradley, A. Mice deficient for p53 are developmentally normal but susceptible to spontaneous tumours. Nature 1992, 356, 215-221. [CrossRef] [PubMed]

34. Jacks, T.; Remington, L.; Williams, B.O.; Schmitt, E.M.; Halachmi, S.; Bronson, R.T.; Weinberg, R.A. Tumor spectrum analysis in p53-mutant mice. Curr. Biol. 1994, 4, 1-7. [CrossRef]

35. Aster, J.C.; Pear, W.S.; Blacklow, S.C. Notch signaling in leukemia. Annu Rev. Pathol. 2008, 3, 587-613. [CrossRef] [PubMed]

36. Dudgeon, C.; Shreeram, S.; Tanoue, K.; Mazur, S.J.; Sayadi, A.; Robinson, R.C.; Appella, E.; Bulavin, D.V. Genetic variants and mutations of PPM1D control the response to DNA damage. Cell Cycle 2013, 12, 2656-2664. [CrossRef]

37. Kleiblova, P.; Shaltiel, I.A.; Benada, J.; Sevcik, J.; Pechackova, S.; Pohlreich, P.; Voest, E.E.; Dundr, P.; Bartek, J.; Kleibl, Z.; et al. Gain-of-function mutations of PPM1D/Wip1 impair the p53-dependent G1 checkpoint. J. Cell Biol. 2013, 201, 511-521. [CrossRef]

38. Bakhshaiesh, T.O.; Armat, M.; Shanehbandi, D.; Sharifi, S.; Baradaran, B.; Hejazi, M.S.; Samadi, N. Arsenic Trioxide Promotes Paclitaxel Cytotoxicity in Resistant Breast Cancer Cells. Asian Pac. J. Cancer Prev. 2015, 16, 5191-5197. [CrossRef] [PubMed]

39. Nahta, R.; Castellino, R.C. Phosphatase magnesium-dependent 1 delta (PPM1D), serine/threonine protein phosphatase and novel pharmacological target in cancer. Biochem. Pharmacol. 2021, 184, 114362. [CrossRef]

40. Mao, J.H.; Li, J.; Jiang, T.; Li, Q.; Wu, D.; Perez-Losada, J.; DelRosario, R.; Peterson, L.; Balmain, A.; Cai, W.W. Genomic instability in radiation-induced mouse lymphoma from p53 heterozygous mice. Oncogene 2005, 24, 7924-7934. [CrossRef] [PubMed]

41. Goh, A.M.; Coffill, C.R.; Lane, D.P. The role of mutant p53 in human cancer. J. Pathol. 2011, 223, 116-126. [CrossRef]

42. Levine, A.J.; Chan, C.S.; Dudgeon, C.; Puzio-Kuter, A.; Hainaut, P. The Evolution of Tumors in Mice and Humans with Germline p53 Mutations. Cold Spring Harb. Symp. Quant. Biol. 2015, 80, 139-145. [CrossRef] [PubMed]

43. Burocziova, M.; Burdova, K.; Martinikova, A.S.; Kasparek, P.; Kleiblova, P.; Danielsen, S.A.; Borecka, M.; Jenikova, G.; Janeckova, L.; Pavel, J.; et al. Truncated PPM1D impairs stem cell response to genotoxic stress and promotes growth of APC-deficient tumors in the mouse colon. Cell Death Dis. 2019, 10, 818. [CrossRef] [PubMed]

44. Gallo-Oller, G.; Vollmann-Zwerenz, A.; Melendez, B.; Rey, J.A.; Hau, P.; Dotor, J.; Castresana, J.S. P144, a Transforming Growth Factor beta inhibitor peptide, generates antitumoral effects and modifies SMAD7 and SKI levels in human glioblastoma cell lines. Cancer Lett. 2016, 381, 67-75. [CrossRef] [PubMed]

45. Fransson, S.; Ostensson, M.; Djos, A.; Javanmardi, N.; Kogner, P.; Martinsson, T. Estimation of copy number aberrations: Comparison of exome sequencing data with SNP microarrays identifies homozygous deletions of 19q13.2 and CIC in neuroblastoma. Int. J. Oncol. 2016, 48, 1103-1116. [CrossRef] 